de Mecánica Computacional

Mecánica Computacional Vol XXV, pp. 1247-1258

Alberto Cardona, Norberto Nigro, Victorio Sonzogni, Mario Storti. (Eds.)

Santa Fe, Argentina, Noviembre 2006

\title{
THE IMPACT OF THE CAVITATION MODEL IN THE ANALYSIS OF MICRO-TEXTURED LUBRICATED JOURNAL BEARINGS
}

\author{
R. Ausas $^{\mathrm{a}}$, G. Buscaglia ${ }^{\mathrm{a}}$, J. Leiva ${ }^{\mathrm{a}}$, M. Jai ${ }^{\mathrm{b}}$, G. Bayada ${ }^{\mathrm{b}}$ and P. $\operatorname{Ragot}^{\mathrm{c}}$ \\ ${ }^{a}$ Centro Atómico Bariloche and Instituto Balseiro, 8400, Bariloche, Argentina e-mail: \\ rfausas@gmail.com,gustavo@cab.cnea.gov.ar \\ ${ }^{\mathrm{b}}$ CNRS-UMR 5208, INSA de LYON, Centre de Mathématiques Bat Leonard de Vinci, F-69622 \\ Villeurbanne, France e-mail: Mohammed.jai@insa-lyon.fr \\ ${ }^{\mathrm{c}}$ RENAULT, Direction de l'Ingenierie Mecanique, Service Mecanique des Solides et Thermique (66151) \\ 92508 RUEIL MALMAISON CEDEX e-mail: patrick.ragot@ renault.com
}

Keywords: Lubrication, Cavitation, Elrod's model, Micro-textures, Journal bearings.

\begin{abstract}
.
In this paper we analyze the impact of the cavitation model on the numerical assessment of lubricated journal bearings. We compare results using the classical Reynolds model and the $p \theta$ model proposed by Elrod to fix the lack of mass conservation of Reynolds' model. Both models give quite similar predictions of load-carrying capacity and friction torque in non-starved conditions, making the Reynolds model to be preferred for its better numerical behavior. Here we report on numerical comparisons of both models in the presence of micro-textured bearing surfaces. We show that in the micro-textured situation the Reynolds model largely underestimates the cavitated area, leading to significant overestimation of the friction torque. This dictates that only mass-conserving models should be used when dealing with micro-textured bearings. Further, we provide numerical details on the algorithms used and apply them to various cases of potential interest for industrial applications.
\end{abstract}




\section{INTRODUCTION}

The use of artificial micro-texturing is becoming popular in oil lubricated devices such as journal bearings because of its potential benefits in terms of friction and wear. This subject is controversial because experimental results exhibiting reductions in the friction coefficient, though not conclusive, contradict the perturbative analysis of (Buscaglia et al., 2005a) which states that small-amplitude textures must increase the friction coefficient. However, the latter theoretical results consider thrust bearings and do not take into account cavitation phenomena, which exists in all journal bearings.

Numerical simulations can account for cavitation phenomena and thus help clarify this issue. There exist two main techniques to model cavitation in lubricated devices: The first is due to Reynolds and reduces to a variational inequality. The second model (Elrod and Adams, 1974) has as main advantage that it enforces mass conservation, which is not satisfied in Reynolds' model. In this paper we consider Elrod's model in the $p \theta$ formulation, thoroughly analyzed mathematically by (Bayada et al., 1998).

It is known that both cavitation models give quite similar results in many practical cases, the two solutions differing just in their prediction of the re-formation boundary. In this article we show that the situation is quite different in micro-textured devices. Since film re-formation occurs at a great number of texture cells, significant differences in the pressure fields predicted by both cavitation models appear. Further, we show that the conservative $p \theta$ formulation predicts a much smaller friction coefficient in the micro-textured case than that predicted by Reynolds' model.

For this purpose, the first part of this paper introduces a finite difference numerical scheme for the conservation of mass equation and the iterative procedure to deal with the non-linearities due to the presence of the cavitation phenomena. We show numerical evidence of the convergence of this method, and then apply it to the comparison of cavitation models in micro-textured journal bearings.

\section{GOVERNING EQUATIONS}

In this section we present the mathematical formulation for the lubrication problem. To this end, in Fig. 1 we show an schematic view of the journal bearing indicating all the relevant parameters and the corresponding bidimensional domain that will be used to write the governing equations.

\subsection{Conservative vs non-conservative formulations}

Cavitation phenomena play an important role in many lubricated devices. It takes place due to a divergent film geometry, at micro-textures (micro-cavitation) or even when feeding is insufficient in the part of a decreasing gap (starvation). As previously mentioned, there are mainly two different techniques to deal with it: the Reynolds model which does not enforce the conservation of mass and Elrod's model which does, the two models differing just in the boundary conditions imposed on the reformation boundaries. An explanation of these two models is presented in what follows.

Initially, in Eq. (1), we present the strong formulation for the two dimensional Reynolds equation (non-dimensionalized and for the stationary case) considering a Newtonian lubricant: 


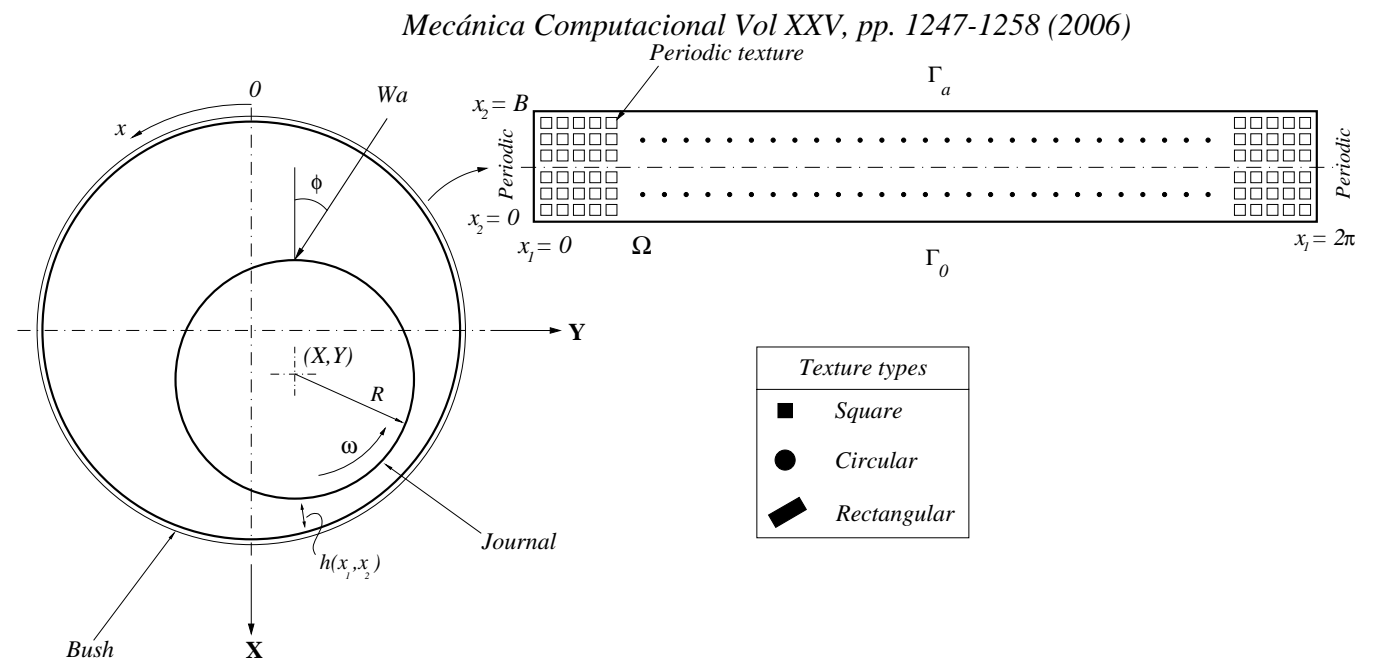

Figure 1: Schematic representation of the journal bearing and the corresponding two dimensional domain.

Find $(p, \theta)$ such that

$$
\begin{aligned}
\nabla \cdot\left(h^{3} \nabla p\right) & =\frac{\partial(\theta h)}{\partial x_{1}} & & p>0 \text { and } \theta=1 \text { in } \Omega_{0} \text { or } p=0 \text { and } 0<\theta<1 \text { in } \Omega^{+} \\
h^{3} \nabla p \cdot \hat{n} & =(1-\theta) h \cos (\hat{n}, \hat{i}) & & \text { on } \Sigma \\
p & =0 & & \text { along } \Gamma_{0} \text { (oil outlet) } \\
p & =p_{a} & & \text { along } \Gamma_{a} \text { (feeding) }
\end{aligned}
$$

In Eq. (1), $h$ represents the distance between the two lubricated surfaces. If $(X, Y)$ is the position of the shaft axis, $h$ is given by:

$$
h\left(x_{1}, x_{2}\right)=1+X \cos \left(x_{1}\right)+Y \sin \left(x_{1}\right)+h_{t}\left(x_{1}, x_{2}\right)
$$

where $h_{t}$ represents the contribution of a texture to the total gap, which we take here to be a periodic function (see Fig. 1), $(\hat{n}, \hat{i})$ is the angle between the unit normal vector to the free boundary $\Sigma$ and the unitary vector along the $x_{1}$ direction, and the sets previously introduced are defined as follows:

$$
\begin{aligned}
\Omega & =(0,2 \pi) \times(0, B) \\
\Gamma_{0} & =\left\{\left(x_{1}, x_{2}\right) \in \partial \Omega / x_{2}=0\right\} \\
\Gamma_{a} & =\left\{\left(x_{1}, x_{2}\right) \in \partial \Omega / x_{2}=B\right\} \\
\Omega^{+} & =\left\{\left(x_{1}, x_{2}\right) \in \partial \Omega / p\left(x_{1}, x_{2}\right)>0\right\} \\
\Omega_{0} & =\left\{\left(x_{1}, x_{2}\right) \in \partial \Omega / p\left(x_{1}, x_{2}\right)=0\right\} \\
\Sigma & =\partial \Omega^{+} \cap \Omega
\end{aligned}
$$

\section{- The Reynolds model}

In the Reynolds model the pressure is the only variable governing the lubrication problem, therefore, $\theta$ does not play a role and is assumed constant and equal to 1 everywhere. This leads 
to the so called Reynolds condition to be applied on any free boundary surface which separates a full-film region from a cavitated one, be it a film ruptured boundary or a film reformation one. The condition reads:

$$
p=\frac{\partial p}{\partial n}=0 \quad \text { on } \Sigma
$$

According to (Bayada and Du Parquet, 1974), this model can be seen in various ways, for instance it can be shown that it reduces to a variational inequality and it can be associated with a well posed problem, but there is no flow in the cavitated area and the equality between output and input flows is not preserved in the model.

\section{- The $p \theta$ model}

In this model, the pressure is not the only unknown governing the cavitation phenomenon. The scalar field $\theta$ is used to account for this, such that the parameter changes between 0 and 1 to separate a full film region $(\theta=1, p>0)$ from a cavitated region $(0<\theta<1, p=0)$. It should be noted that condition given in Eq. (4) holds on any film-rupture boundary, but this is not the case on film-reformation boundaries, for which the appropiate condition to be considered as indicated in Eq. (1) is $h^{3} \nabla p \cdot \hat{n}=(1-\theta) h \cos (\hat{n}, \hat{i})$ (Bayada and Du Parquet, 1974). In this case, by applying the mass conservation equation in the variational sense, a possible jump of $\theta$ and $\partial p / \partial n$ through the free boundary is allowed. Besides, it allows for micro-cavitation and starvation to take place.

Regarding the physical interpretation of this scalar field $\theta$, it can be viewed as a saturation function (Bayada and Chambat, 1986) or a fractional film content (Elrod and Adams, 1974) or the result of the homogenization of the oil fingers through the height of the gap (Jakobson and Floberg, 1957).

\section{NUMERICAL DETAILS}

\subsection{General setting}

The first step in the numerical formulation is the spatial discretization of the equations by means of a finite difference approach. Considering a discretization of the computational domain $\Omega$ into $I$ points in the $x_{1}$ direction and $J$ points in the $x_{2}$ direction, the following stencil, which involves both unknown fields $p$ and $\theta$, is obtained for the $p \theta$ model at the $(i, j)$-th node:

$$
\begin{array}{r}
s_{i}^{j} p_{i+1}^{j}-\left(s_{i}^{j}+s_{i-1}^{j}\right) p_{i}^{j}+s_{i-1}^{j} p_{i-1}^{j}+q^{2}\left\{s_{i}^{j+1} p_{i}^{j+1}-\left(s_{i}^{j+1}+s_{i}^{j}\right) p_{i}^{j}+s_{i}^{j+1} p_{i}^{j-1}\right\}= \\
=\left(c_{i}^{j}-c_{i-1}^{j}\right) \Delta x_{1}
\end{array}
$$

where

$$
s_{i}^{j}=\theta_{i}^{j}\left(h_{i}^{j}\right)^{3} \quad c_{i}^{j}=\theta_{i}^{j} h_{i}^{j} \quad q=\frac{\Delta x_{1}}{\Delta x_{2}}
$$

and where the following definitions for the fluxes along the $x_{1}$ and $x_{2}$ directions have been assumed: 


$$
\left.\frac{\partial p}{\partial x_{1}}\right|_{i} ^{j}=\left.\frac{p_{i+1}^{j}-p_{i}^{j}}{\Delta x_{1}} \quad \frac{\partial p}{\partial x_{2}}\right|_{i} ^{j}=\frac{p_{i}^{j}-p_{i}^{j-1}}{\Delta x_{2}}
$$

\subsection{Treatment of the non-linearities}

The equations arising from the Reynolds model can be solved by using standard finite element or finite difference methods. In this case, the boundary condition expressed by Eq. (4) can be imposed by means of a penalization approach, in which the resulting system of equations is modified by substituting on-the-fly, zero in any negative value of the pressure as done by Buscaglia et al. (2005b). Unfortunately, we have not been able to find a robust extension of this penalization approach to the $p \theta$ formulation in micro-textured configurations. Notice that the smallness of the textures requires very fine meshes, leading to huge systems of nonlinear equations that can only be solved with very robust algorithms. The method we have chosen deals quite well with the non-linearities present in both models. It is a Gauss-Seidel iterative process, in which no assembly of any linear system is needed. In particular this approach is much more convenient for the $p \theta$ model due to the stiffness of the discretized equations, and it could be used without problems in the Reynolds model as a particular case.

To formulate the iterative algorithm, the pressure $p$ and the $\theta$ field at each node are separately obtained from the stencil given in Eq. (5) as functions of all the remaining quantities in it. This is not a problem, because in the cavitated area the pressure is known $(p=0,0<\theta<1)$ while in the non-cavitated region $\theta$ is known $(\theta=1, p>0)$. This leads to the following numerical scheme:

$$
\text { Find }\left(p_{i}, \theta_{i}\right) i=1,2, \ldots, I \times J \text { such that }
$$

$$
\left(p_{i}, \theta_{i}\right)=\lim _{n \rightarrow \infty}\left(p_{i}{ }^{n}, \theta_{i}{ }^{n}\right),\left(p_{i}{ }^{0}, \theta_{i}{ }^{0}\right)=\left(p_{i}{ }^{G}, \theta_{i}{ }^{G}\right)
$$

where $\left(p_{i}{ }^{G}, \theta_{i}{ }^{G}\right)$ are initial nodal guess values and each new iterate is built according to:

$$
\begin{aligned}
p_{i}{ }^{n} & =\omega_{p} P_{i}^{n}+\left(1-\omega_{p}\right) p_{i}^{n-1} \\
\theta_{i}{ }^{n} & =\omega_{\theta} \Theta_{i}^{n}+\left(1-\omega_{\theta}\right) \theta_{i}^{n-1}
\end{aligned}
$$

and where $\omega_{p}$ and $\omega_{\theta}$ are the relaxation parameters for the pressure and the $\theta$ field respectively and the functions $P_{i+1}^{n}$ and $\Theta_{i+1}^{n}$ are defined generically in the following way:

$$
\left(P_{i+1}^{n}, \Theta_{i+1}^{n}\right)=(f(\tilde{p}, \tilde{\theta}), g(\tilde{p}, \tilde{\theta}))
$$

where we mean by $(\tilde{p}, \tilde{\theta})$ all the possible variables that are present in the arguments of $f$ and $g$, including values of the pressure and $\theta$ field at iterations $n$ and $n-1$.

For the Reynolds model, the same scheme is used, with the only difference that the field $\theta$ is taken equal to 1 everywhere and if the pressure becomes negative, this is automatically clipped, setting it to 0 in order to impose the Reynolds condition. 
4 NUMERICAL RESULTS

The aim of this section is to present a comparison of the two models previously described. As mentioned, the models predict quite similar results in many practical cases, but this is not the situation when micro-textures are present, since film reformation occurs at a great number of texture cells. The use of textures in industrial applications makes the determination of a suitable model to correctly evaluate their performance of major importance.

\subsection{Preliminary definitions}

Two essential quantities of journal-bearing behavior are the load carrying capacity and the friction torque, given by

$$
\begin{gathered}
W_{X}=\int_{\Omega} p \cos \left(2 \pi x_{1}\right) d \Omega \quad W_{Y}=\int_{\Omega} p \sin \left(2 \pi x_{1}\right) d \Omega \\
T=\int_{\Omega^{+}}\left(\frac{1}{h}+3 h \frac{\partial p}{\partial x_{1}}\right) d \Omega
\end{gathered}
$$

As we will see, the integration over $\Omega^{+}$instead of $\Omega$ in the definition of $T$ is of fundamental importance to understand the differences in the prediction of this quantity by the two models. In the definitions of $W_{X}$ and $W_{Y}$ this does not play a role because the integrand contains the pressure, which is zero outside $\Omega^{+}$.

The friction coefficient $T$ is evaluated at a certain equilibrium position $(X, Y)$ corresponding to a given applied load $W_{a}$ on the journal. This equilibrium position is found by performing a Newton-Raphson iteration process on the following equilibrium condition:

$$
W_{a} \cos (\phi)+W_{X}(X, Y)=0 \quad W_{a} \sin (\phi)+W_{Y}(X, Y)=0
$$

where $\phi$ is the angle of the applied load as indicated in Fig. 1.

\subsection{Simulations}

The main interest regards the use of textures to improve the bearing performance. Experimental and theoretical results in different types of lubricated devices including journal bearings exhibit improvements in their static and dynamic characteristics, such as reductions in the friction coefficient (see for instance (Etsion and Halperin, 2002; Ryk et al., 2002; Brizmer et al., 2003; Kovalchenko et al., 2004)) or increments in the stiffness and damping coefficients, see for example Kato and Obara (1996). Besides, the textures have some other benefits, like the possibility of using them as a reservoir for extra lubricant or even their ability to trap solid particles present in it.

In this work we are interested in the effects of micro textures with a uniform distribution troughout the domain. The aim will be in comparing the bearing performace with the two models in various cases: First we will present typical results of the pressure and the $\theta$ field in a journal with and without textures. Then, we will study the effect of the various texture's parameters, such as the the size, the texture depth, the fraction area and shape.

In Figs. 2 and 3 we show the pressure and the $\theta$ fields corresponding to a smooth journal bearing obtained by means of the $p \theta$ model. This calculation has been done with a mesh containing $750 \times 75$ cells. 


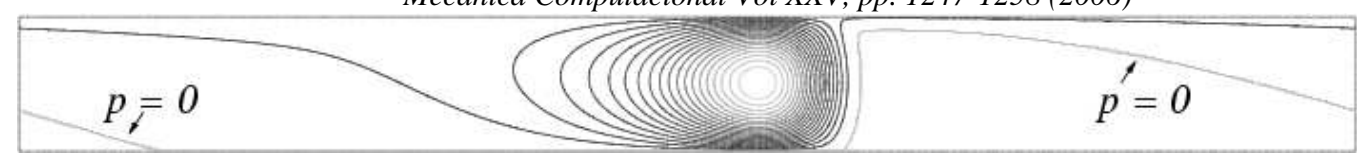

Figure 2: Pressure field obtained with the $p \theta$ model for a smooth journal bearing. Minimum $=0$ (light gray), Maximum $=0.128$ (dark gray).

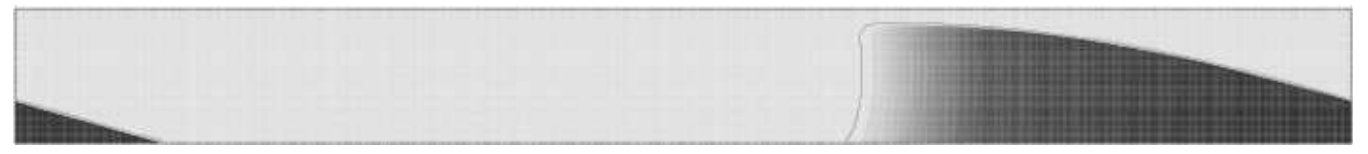

Figure 3: $\theta$ field obtained with the $p \theta$ model for a smooth journal bearing. Minimum $=0.0$ (dark gray), Maximum $=1.0$ (light gray).

In Fig. 2 the isoline corresponding to $p=0$ has been indicated as well as the isoline corresponding to $\theta=1$ in Fig. 3 .

For the cases we will study, the texture will have square shape and will be defined by the following parameters:

- Number of textures along the $x_{1}$ and $x_{2}$ directions $\rightarrow N_{1}$ and $N_{2}$

- Texture Depth $\rightarrow h_{t 0}$

- Fraction area $\rightarrow s$

Additionally, the dimensionless bearing width $B$ and feeding pressure $p_{a}$ will be taken as 0.1 and 0.0075 respectively.

In a smooth case the difference in the pressure field as predicted by the two models is not important, but when the texture is introduced, significative differences can be observed. In Fig. 4 we show a comparison between the two models for the one dimensional pressure field along a cross section of constant $x_{2}$ when different texture arrays are considered. As it can be seen, while the Reynolds model predicts a pressure different than zero at the locations of the individual textures, the $p \theta$ model predicts a pressure exactly equal to zero at those regions. This can be understood looking at the $\theta$ field obtained with the $p \theta$ model, where the value of $\theta$ at those places lies between 0 and 1 (see Fig. 5).

Finally, we compare the bearing performance obtained with the two models by looking at the friction torque (see Eq. (10)). On the one hand, we consider a textured bearing with an array of $N_{1} \times N_{2}=50 \times 5$ square textures and on the other hand, a smooth bearing. For these simulations a mesh containing $750 \times 75$ discretization cells is used which leads to a significative computational effort.

First, in Fig. 6 we show the friction torque as a function of the applied load for different values of the texture depth $h_{t 0}$ and a fixed value of $20 \%$ for the fraction area $s$. The results for the $p \theta$ model are shown on the left and on the right the results corresponding to the Reynolds model.

Then, in Fig. 7 we show the comparison when the texture depth $h_{t 0}$ is kept constant in 0.45 and the fraction area $s$ is changed.

As it can be seen, both models give similar results for the smooth bearing, however, there is a significative difference between them for the textured cases. In Fig. 8, we can appreciate this 
(a) Smooth

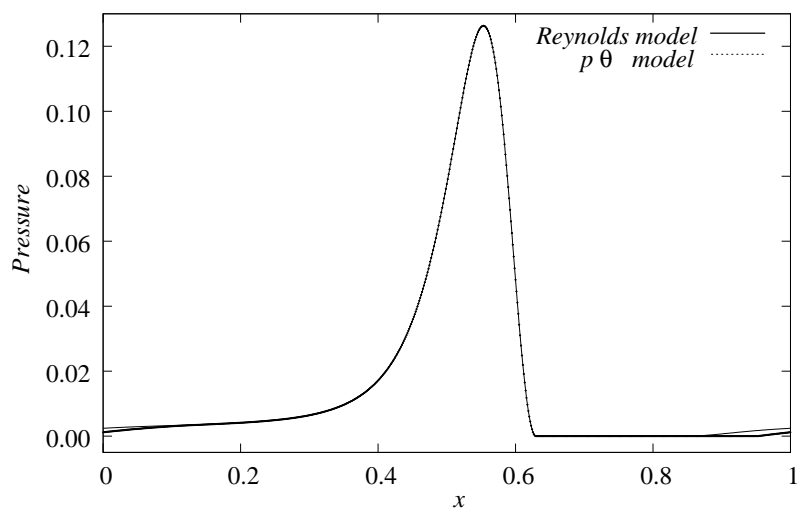

(c) Array $100 \times 10$

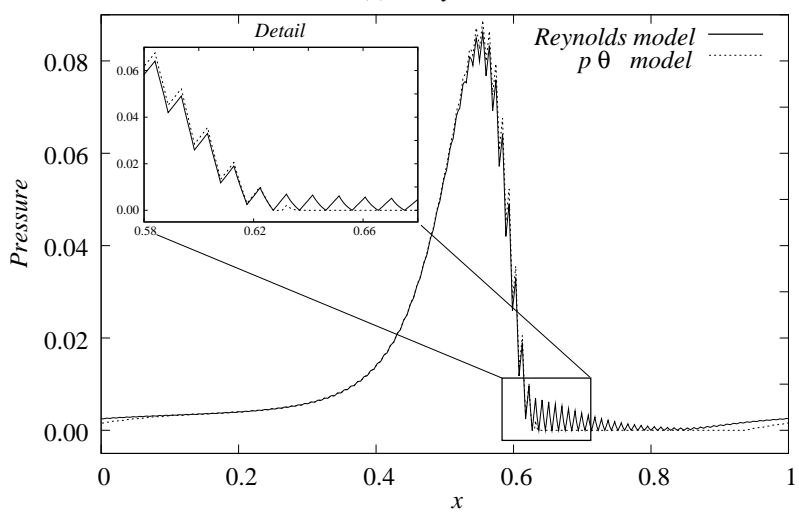

(b) Array 50x5

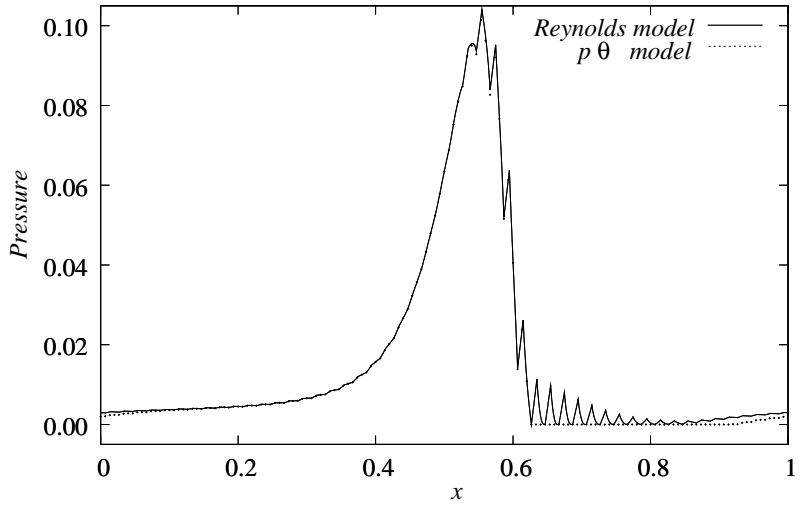

(d) Array 200x20

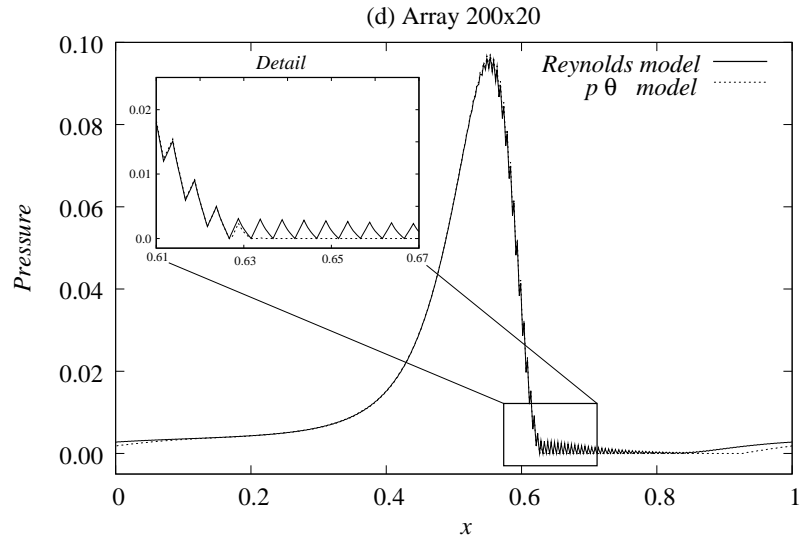

Figure 4: Comparison between the two models of the one dimensional pressure profile along a cross section of constant $x_{2}$. (a) Smooth case (Mesh: $500 \times 50$ ), (b) Array $50 \times 5$ (Mesh: $750 \times 75$ ), (c) Array $100 \times 10$ (Mesh: $1250 \times 125)$, (d) Array $200 \times 20$ (Mesh: $2500 \times 250$ ).
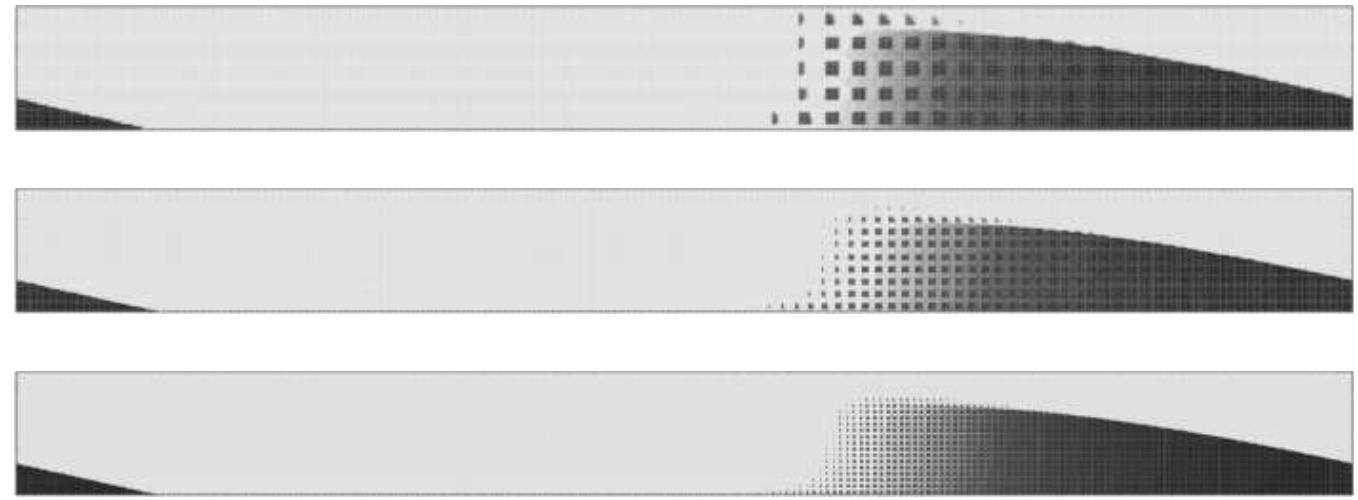

Figure 5: $\theta$ field obtained with the $p \theta$ model for different arrays of textures considered in Fig. 4. Top: Array $50 \times 5$, Middle: Array $100 \times 10$, Bottom: Array $200 \times 20$. Minimum $=0.0$ (dark gray), Maximum $=1.0$ (light gray). 

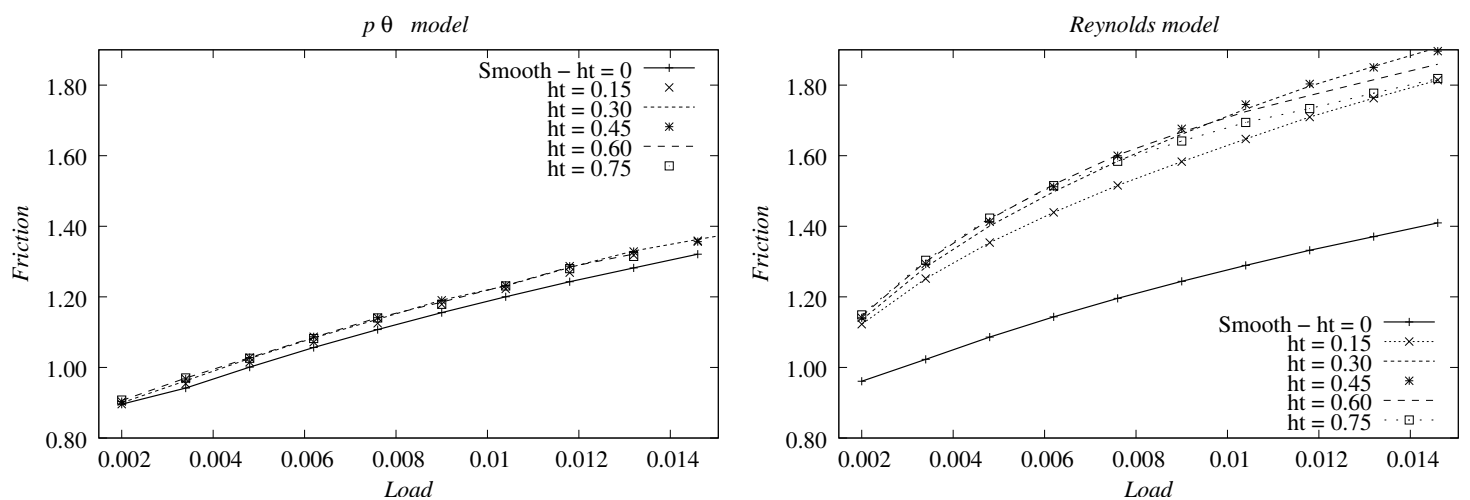

Figure 6: Friction force $T$ as a function of the applied load $W_{a}$ for different values of the texture depth $h_{t 0}$. Fraction area $s=20 \%$. Left: $p \theta$ model, Right: Reynolds model.
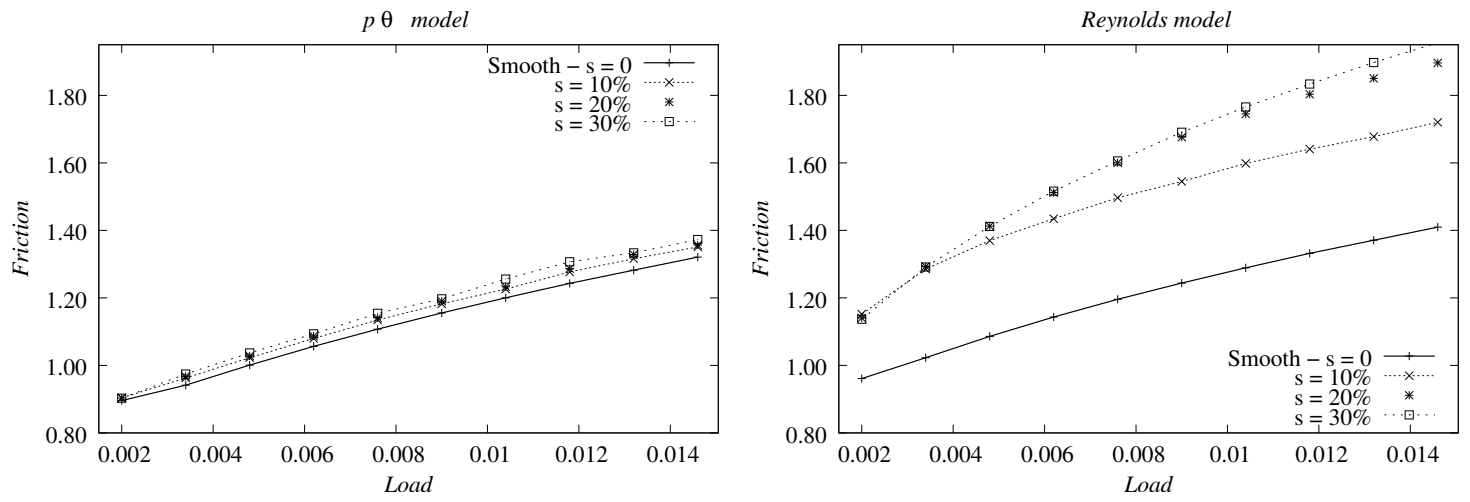

Figure 7: Friction force $T$ as a function of the applied load $W_{a}$ for different values of the fraction area $s$. Texture depth $h_{t 0}=0.45$. Left: $p \theta$ model, Right: Reynolds model. 
when ploting the friction torque as a function of the texture depth when the parameter $s=20 \%$. As we can see for all these cases, the impact on the friction torque due to the presence of the texture is much more important as predicted by the Reynolds model.
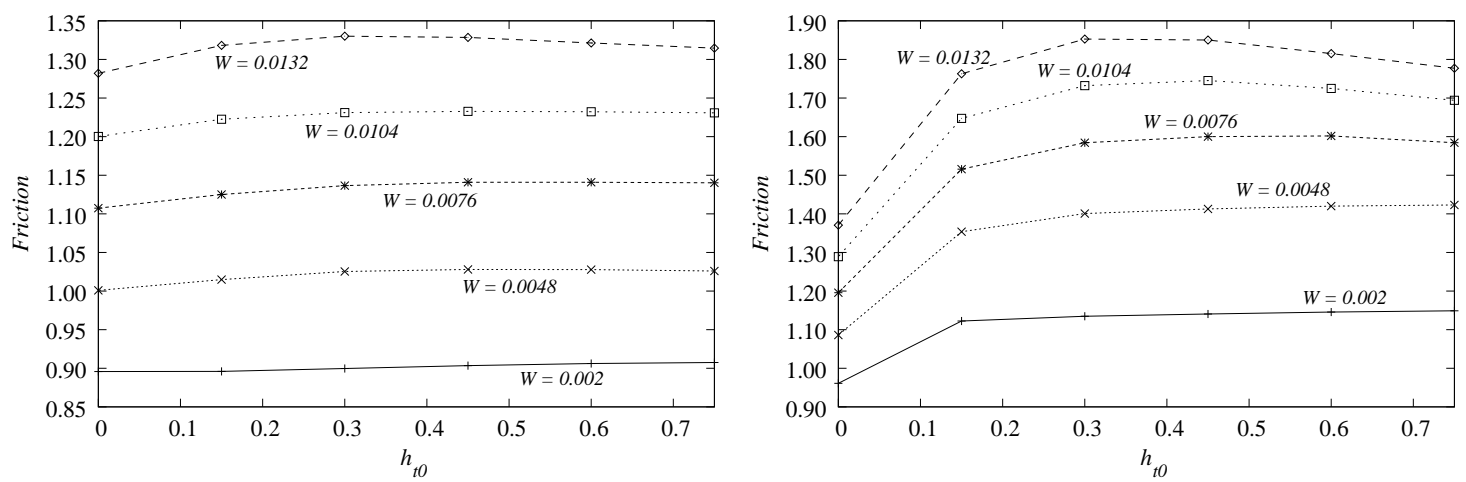

Figure 8: Friction force $T$ as a function of the texture depth $h_{t 0}$ for different values of the applied load $W_{a}$. Fraction area $s=20 \%$. Left: $p \theta$ model, Right: Reynolds model.

Similar results to those presented have been obtained for other types of textures. As an example, in Table 1 we compare the friction torque in different cases including square, circular and rectangular textures in a periodic array of $N_{1} \times N_{2}=50 \times 5$ (see Fig. 1). In all the cases the texture depth as well as the fraction area are kept constant in 0.3 and $20 \%$ respectively. The two different terms known as the Couette and Poiseuille components that define the friction torque (see Eq. (10)) as well as the number of discretization cells used for the simulations in each case are also included.

Table 1: Friction force $T$ and its components corresponding to the different types of textures presented in Fig. 1. For all the cases the texture depth is taken as $h_{t}=0.3$ and the fraction area $s=20 \%$.

\begin{tabular}{|l|c|c|c|c|}
\hline Texture type & Overall Friction & Couette $(=1 / h)$ & Poiseuille $\left(=3 h \partial p / \partial x_{1}\right)$ & Mesh \\
\hline Smooth & 1.201 & 1.131 & 0.070 & $500 \times 50$ \\
Square & 1.223 & 1.101 & 0.122 & $750 \times 75$ \\
Circular & 1.235 & 1.094 & 0.141 & $1500 \times 150$ \\
Rectangular & 1.244 & 1.117 & 0.127 & $1250 \times 125$ \\
\hline
\end{tabular}

In principle, taking pure hidrodynamic arguments into account, the utilization of textures seems like an attractive idea, because it tends to decrease the first term of the friction torque (the Couette component) which is proportional to $\frac{1}{h}$. However, due to the presence of the texture the system assumes a new equilibrium position corresponding to the given applied load and besides, the second term of the friction torque (the Poiseuille component) is one proportional to $h$ and the pressure gradient along the $x_{1}$ direction.

Finally, it should be noted, the significantly lower value for the friction force predicted by the $p \theta$ model compared to the Reynolds model. This situation, come out from the fact that film 
reformation occurs at a great number of texture cells and while Reynolds formulation predicts small values (different than zero) for the pressure in some of these texture cells, the $p \theta$ model predicts values for $\theta$ that are smaller than one at those regions, and as a consequence a pressure exactly equal to zero. This is clearly shown in Fig. 4.

\section{CONCLUSIONS}

In this paper we analize the use of two different cavitation models for the numerical evaluation of micro-textured journal bearings: the Reynolds model and the $p \theta$ model, the two models differing in the boundary condition which is imposed on the reformation boundaries. A numerical algorithm based on a Gauss-Seidel iterative process has been developed to deal with the non-linear character of the resulting discretized equations in both models.

Various cases have been studied considering different values for the texture size, depth, fraction area and shape and it has been seen that the Reynolds model largely overestimates the friction torque as compared to the $p \theta$ formulation. It has been seen that this is due to an underestimation of the cavitation area. These observations show that a mass-conservative formulation is needed in order to correctly evaluate the journal bearing performance when micro-textured surfaces are present.

Future work includes a more extensive analysis on the range of applied loads, since evidence of friction torque improvements due to the micro-textures has been observed for very low values of the applied load. Further, the analysis of journal bearings under dynamic loading in cases with micro-textured moving surfaces is under current development.

\section{ACKNOWLEDGMENTS}

Work partially supported by Grant NOS. PICT 12-9848 (FONCYT, Argentina) and A03E01 (ECOS-SECYT). G.C.B. also belongs to CONICET (Argentina).

\section{REFERENCES}

G. Bayada and M. Chambat. The transition between the stokes equation and the reynolds equation: A mathematical proof. Appl. Math. Opt., 14, 1986.

G. Bayada, M. Chambat, and C. Vazquez. Characteristics method for the formulation and computation of a free boundary cavitation problem. Journal of Computational and Applied mathematics, 98, 1998.

G. Bayada and J. Du Parquet. The influence of operating parameters on the cavitation in a dynamically loaded journal bearing. 1 st LEEDS LYON Symposium on Cavitation and Related Phenomena in Lubrication, I.M.E., 14, 1974.

V. Brizmer, Y. Kligerman, and I. Etsion. A laser surface textured thrust bearing. Tribology Transactions, 46, 2003.

G.C. Buscaglia, S. Ciuperca, and M. Jai. The effect of periodic textures on the static characteristics of thrust bearings. Journal of Tribology, 127, 2005a.

G.C. Buscaglia, A.F. Quiroga, G. Bayada, and M. Jai. Sensitivity of lubricated devices to changes in their macro/microscopic shape. Proceedings of the 6th World Congresses of Structural and Multidisciplinary Optimization, Rio de Janeiro, $2005 \mathrm{~b}$.

H. G. Elrod and M Adams. A computer program for cavitation. 1st LEEDS LYON Symposium on Cavitation and Related Phenomena in Lubrication, I.M.E., 103, 1974.

I. Etsion and G. Halperin. A laser surface textured hydrostatic mechanical seal. Tribology Transactions, 45, 2002. 
B. Jakobson and L. Floberg. The finite journal bearing considering vaporization. Tran. Chalmers University of Technology, 103, 1957.

T. Kato and S. Obara. Improvement in dynamic characteristics of circular journal bearings by means of longitudinal microgrooves. Tribology Transactions, 39, 1996.

A. Kovalchenko, O. Ajayi, A. Erdemir, and Etsion I. Fenske, G. The effect of laser texturing of steel surfaces and speed-load parameters on the transition of lubrication regime from boundary to hydrodynamic. Tribology Transactions, 47, 2004.

G. Ryk, Y. Kligerman, and I. Etsion. Experimental investigation of laser texturing for reciprocating automotive components. Tribology Transactions, 45, 2002. 